Research Article

\title{
Lignosulfonates as Fire Retardants in Wood Flour-Based Particleboards
}

\author{
Stefania Angelini (D, ${ }^{1}$ Aitor Barrio, ${ }^{2}$ Pierfrancesco Cerruti (iD, ${ }^{3}$ Gennaro Scarinzi, ${ }^{1}$ \\ Javier Garcia-Jaca, ${ }^{2}$ Davide Savy, ${ }^{4}$ Alessandro Piccolo, ${ }^{4}$ and Mario Malinconico ${ }^{1}$ \\ ${ }^{1}$ Institute for Polymers, Composites and Biomaterials, Via Campi Flegrei 34, Pozzuoli, Naples 80078, Italy \\ ${ }^{2}$ Tecnalia Research \& Innovation, Area Anardi 5, Azpeitia, Guipùzcoa E-20730, Spain \\ ${ }^{3}$ Institute for Polymers, Composites and Biomaterials (IPCB-CNR), Via Previati 1/E, 23900 Lecco, Italy \\ ${ }^{4}$ Interdepartmental Research Centre of Nuclear Magnetic Resonance for the Environment, Food Processing and New \\ Materials (CERMANU), Via Università 100, Portici 80055, Italy
}

Correspondence should be addressed to Stefania Angelini; stefaniangelini@gmail.com

Received 14 January 2019; Revised 9 May 2019; Accepted 7 June 2019; Published 11 July 2019

Academic Editor: De-Yi Wang

Copyright (c) 2019 Stefania Angelini et al. This is an open access article distributed under the Creative Commons Attribution License, which permits unrestricted use, distribution, and reproduction in any medium, provided the original work is properly cited.

\begin{abstract}
Wood flour particleboards (WFP), like other wood-based items, require the addition of fire retardants (FRs) to reduce their high flammability. In this work, a waste lignosulfonate (CaLS) from paper mill is used as a low-cost FR to reduce WFP flammability. CaLS is purified by dialysis and the dialysed lignosulfonate (LD) is used, alone or combined with ammonium polyphosphate (APP), as a FR additive in the preparation of urea-formaldehyde WFP. The fire behaviour of the modified WFPs is studied by cone calorimetry. The use of $15 \mathrm{wt} . \% \mathrm{LD}$ reduces the peak of heat release rate (HRR) and total smoke production by $25 \%$, also increasing char formation. HRR peak is further reduced up to $40 \%$, if APP is introduced in the formulation. This work discloses a viable and cost-effective strategy for improving the fire retardancy performance of WFP by partial replacement of a commercial FR with a fully renewable additive, isolated through a green and cost-effective process.
\end{abstract}

\section{Introduction}

Lignocellulosic biomass is the most abundant renewable material and is widely employed for industrial and agricultural purposes. Its use involves the production of a great amount of lignocellulosic waste that is usually landfill disposed or burnt as an energy source. A non-negligible portion of this byproduct comes from pulp and paper mills and bioethanol production [1].

In particular, pulp and paper industry produces lignosulfonates (LS) through sulphite process, in which lignin is removed from wood chips by means of sulphite salts to isolate almost pure cellulose fibers [2]. The sulphite treatment involves the cleavage of ether linkages between the phenylpropanoid moieties, and the introduction of sulfonic acid groups on the aliphatic chains of lignin, resulting in the formation of water soluble and surface-active LS [3]. The latter are used as dispersants [4], emulsion stabilizers [5], flocculating agents [6], and source of low-molecular weight aromatic chemicals $[7,8]$. Alongside these applications, waste LS have been applied as reinforcing filler or additive in polymer composites and related materials, providing benefits in terms of production costs, potential biodegradability, and reinforcement features [9-12]. However, notwithstanding these applications, only $1 \%$ of the lignosulfonates produced all over the world are used. Therefore, new routes for the exploitation of these coproducts are desirable [3].

Another research approach that has been preliminarily investigated deals with the use of lignins and lignosulfonates as fire retardant (FR) charring additives. One of the most 
common methods to increase fire resistance of highly flammable materials consists in the incorporation of FRs, i.e., additives able to interfere with combustion [13]. The use of FRs was reported in thermoplastic polymers [14, 15] as well as in wood $[16,17]$ and wood-based products such as particleboards and fiberboards [18-20]. Most FRs are inorganic, halogenated, or phosphorous compounds, such as hexabromocyclododecane or ammonium polyphosphate (APP). They are usually effective as flame suppressors, but they also show high toxicity, as in the case of halogenated FRs, or other remarkable drawbacks related to their preparation. APP is considered one of the most sustainable commercial FRs, due to its low toxicity. However, its synthesis involves harmful chemicals such as phosphorous pentoxide, which is difficult to handle [21]. Moreover, the use of FRs leads to a cost increase of the final product. On the other side, LS, similarly to other lignins, are potential substitutes of commercial FRs due to their renewable character, high carbon content, and aromatic structure. The last two features allow to reduce the combustion rate during a fire, promoting the formation of an insulating char that preserve the material from further burning [22-24]. Such effect was evidenced in fire tests performed on lignin-modified polypropylene/wood composites [25]. In addition, LS are easily available on a large scale and cost-effective when compared to commercial FRs, e.g., calcium LS is about five times cheaper than APP [26].

The high charring tendency of lignins during combustion [27] make them potential substitutes of traditional fire suppressors. Their use in this field dates back to the eighties [28]; however, over the years, much more interest has been devoted to lignin rather than lignosulfonates, and the potentiality of the latter in fire retardancy has not been fully explored yet. In addition, although the published works report an improvement in flame suppression properties, the applicability of the proposed methods remains hampered by the difficult procedures for preparing samples. Indeed, the most typical are based on layer-by-layer deposition of lignin on the polymeric substrates [9] or its chemical modification $[29,30]$. Based on these issues, nowadays, the attention has been moving towards the use of nonmodified lignins in order to reduce both the amount of chemicals and the cost of the additive [31].

Following this path, in this work calcium LS (CaLS) was purified and used as an additive in wood flour particleboards (WFP) bound with urea-formaldehyde (UF) resin. The aim of the paper is to isolate the lignin component of CaLS by a simple and green procedure and to directly test it as a fire suppressor to improve the fire performances of the WFP in a technologically feasible and cost-effective way. Raw CaLS was purified through dialysis against water, affording a carbohydrate-free dialysed lignin (LD). Then, the effect of LD was evaluated by analysing fire performance, thermal degradation behaviour, and mechanical properties of the prepared composites. For the sake of comparison, samples modified with the commercial fire retardant APP, as well as with both APP and LD, were characterized. The main properties of the obtained composites were analysed in the perspective of using them in furniture or green building applications.

\section{Materials and Methods}

2.1. Materials. CaLS was kindly provided by the Burgo Group, Italy. Wood flour (WF) (particle size $<400 \mu \mathrm{m}$ ) and urea-formaldehyde (UF) resin Q110T were provided from Beologic, Belgium, and KRONOSPAN Chemicals, respectively. Fire retardant Exolit AP 420 aqueous solution (45 wt.\% solid content of a short-chain ammonium polyphosphate (APP)) was purchased from Clariant. Milli-Q water was used to perform the dialysis of CaLS solutions. Concentrated sulfuric acid (95-98\%) was purchased from Sigma-Aldrich.

\subsection{Lignosulfonates Purification and Characterization}

2.2.1. Dialysis. CaLS was submitted to filtration and dialysis to remove hemicelluloses, simple sugars, and inorganic contaminants from the feedstock. Dialysis was performed using the Spectra/Por 3 tubes of regenerated cellulose with a molecular mass cut-off of $3.5 \mathrm{kDa}, 29 \mathrm{~mm}$ diameter, and $45 \mathrm{~mm}$ flat width. CaLS aqueous solution at $10 \mathrm{wt} . \%$ concentration was filtered on paper (porosity 1-11 $\mu \mathrm{m}$ ), and the brown, cloudy filtrate was dialysed against water for $12 \mathrm{~h}$. After the dialysis, the solution was evaporated to dryness under reduced pressure, at $80^{\circ} \mathrm{C}$. The obtained product, referred to as $\mathrm{LD}$, was further oven-dried under vacuum at $60^{\circ} \mathrm{C}$.

2.2.2. Chemical Characterization. Solid-state ${ }^{13} \mathrm{C}$ CP MASNMR characterization was performed on CaLS and LD. Measurements were carried out using a Bruker Avance II 400 spectrometer operating at $100.47 \mathrm{MHz}$. Samples were spun at $10 \mathrm{kHz}$ in $4 \mathrm{~mm}$ zirconium oxide rotors. Spectra were collected using a single pulse excitation sequence with a ${ }^{13} \mathrm{C} 90^{\circ}$ pulse width of $3.2 \mathrm{~s}$, a recycle delay of $2 \mathrm{~s}$, and a contact time of $2 \mathrm{~ms}$ by averaging 16,384 scans.

The dialysate was analysed before and after acid hydrolysis with $\mathrm{H}_{2} \mathrm{SO}_{4}$ [32] by a Thermo Scientific Dionex Ultimate 3000 High-Performance Liquid Chromatography (HPLC) device equipped with an Aminex Bio-Rad HPX87H column and a refractive index detector.

2.2.3. Thermal Analysis. Thermogravimetric investigation (TGA) was carried out by means of a PerkinElmer Pyris Diamond TG/SDTA thermobalance with alumina pans using about $5 \pm 0.5 \mathrm{mg}$ of CaLS or LD. Measurements were carried out in nitrogen atmosphere under a flow rate of $30 \mathrm{~mL} \mathrm{~min}^{-1}$. Each sample was analysed according to the following thermal program: heating from 30 to $90^{\circ} \mathrm{C}$ at $10^{\circ} \mathrm{C} \mathrm{min}^{-1}$, isotherm at $90^{\circ} \mathrm{C}$ for $20 \mathrm{~min}$, and heating from 90 to $500^{\circ} \mathrm{C}$ at $10^{\circ} \mathrm{C} \mathrm{min}^{-1}$. A temperature of $5 \%$ weight loss $\left(T_{5 \%}\right)$, temperature of maximum decomposition rate $\left(T_{\max }\right)$, and char yield at $500^{\circ} \mathrm{C}\left(\mathrm{Char}_{500^{\circ} \mathrm{C}}\right)$ were measured. Tests were carried out in duplicate.

\subsection{Particleboard Preparation and Characterization}

2.3.1. Particleboard Preparation. Particleboards were prepared using WF as filler, UF resin as binder, and LD and APP as FR additives. UF was used at 15 wt.\% with respect to the total solid amount $(150 \mathrm{~g})$. First, WF was soaked in about $380 \mathrm{~g}$ of water. Then UF, LD, and APP were added, 
and the sludge was vigorously stirred in order to homogenize it. The prepared formulations were poured in vessels and evaporated in an oven at $60^{\circ} \mathrm{C}$ overnight. Next, the samples were ground, sieved (using a sieve of $500 \mu \mathrm{m}$ porosity), and vacuum-dried in oven at $50^{\circ} \mathrm{C}$ for $1 \mathrm{~h}$. Finally, particleboards were obtained through compression moulding by means of a Cortazar Especial platen press. The compounds were preheated for $1 \mathrm{~min}$ at $220^{\circ} \mathrm{C}$, then pressed for $3 \mathrm{~min}$ at $100 \mathrm{bar}$, and cooled down to room temperature. The particleboard codes and compositions on a dry basis are reported in Table 1.

2.3.2. Mechanical Tests. Flexural tests were performed on $7.00 \times 0.50 \times 1.00 \mathrm{~cm}^{3}$ specimens cut from the particleboard plates by means of an Instron model 4505 dynamometer, at a deformation speed of $1 \mathrm{~mm} \mathrm{~min}^{-1}$ with a span of $48 \mathrm{~mm}$. Four specimens were used for each formulation.

2.3.3. Thermal Analysis. TGA investigation was carried out on particleboards by means of a Mettler-Toledo TG-SDTA 851 thermobalance equipped with a differential thermal analyser, using alumina pans under nitrogen flow $\left(35 \mathrm{~mL} \mathrm{~min}^{-1}\right)$. After a rapid heating from 25 to $100^{\circ} \mathrm{C}$ at $20^{\circ} \mathrm{C} \mathrm{min}^{-1}$ to remove absorbed moisture, the samples were kept in isothermal conditions for $30 \mathrm{~min}$ and then heated up to $700^{\circ} \mathrm{C}$ at

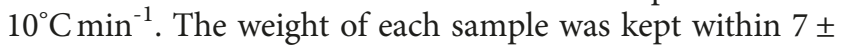
$1 \mathrm{mg}$. A temperature of $5 \%$ weight loss $\left(T_{5 \%}\right)$, temperature of maximum decomposition rate $\left(T_{\max }\right)$, and char yield at $700^{\circ} \mathrm{C}\left(\right.$ Char $\left._{700^{\circ} \mathrm{C}}\right)$ were measured.

2.3.4. Cone Calorimeter Tests. The particleboards (density around $0.6 \mathrm{~g} \mathrm{~cm}^{-3}$ ) were cut to obtain $10 \times 10 \times 0.5 \mathrm{~cm}^{3}$ plates. All the plates were stored in a conditioning chamber at $23^{\circ} \mathrm{C}$ and $48 \%$ relative humidity for a week before being tested. Combustion experiments were performed on the plates by means of a cone calorimeter (Fire Testing Technology, FTT), at an incident heat flux of $35 \mathrm{~kW} \mathrm{~m}^{-2}$ and a distance of $25 \mathrm{~mm}$ from the heater. The experiments were carried out in an $800 \mathrm{~s}$ time span. Heat release rate (HRR,

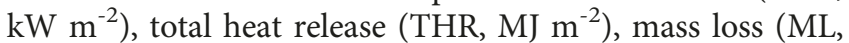
$\%$ ), total smoke production (TSP, $\mathrm{m}^{2}$ ), and specific extinction area (SEA, $\mathrm{m}^{2} \mathrm{~kg}^{-1}$ ) were measured. All tests were performed in triplicate and in accordance with ISO 5660-1:2002.

\section{Results and Discussion}

3.1. Purification and Characterization of Lignosulfonates. Characterization of CaLS was performed by SEM (see Fig. S1 in Supplementary Materials) and ${ }^{13} \mathrm{C}$ CP MAS-NMR spectroscopy. NMR spectra are reported in Figure 1(a), while resonance assignments and detailed discussion of NMR signals are provided in Supplementary Materials (see Table S1 and chemical analysis of lignosulfonates). From Figure 1(a), CaLS spectrum (blue trace) shows resonances ascribed to the main lignocellulosic macromolecular components, namely, lignin and hemicellulose polysaccharides. In order to remove the polysaccharide contaminant, CaLS was submitted to dialysis against water. The spectrum of the dialysed sample (LD) (Figure 1(a), red trace) exhibits only the typical signals of lignin materials. Resonances centred at
TABle 1: Particleboard codes and compositions (wt.\%).

\begin{tabular}{lccc}
\hline Sample & WF & APP & LD \\
\hline WF100 & 100 & - & - \\
WF95-LD5 & 95 & - & 5 \\
WF85-LD15 & 85 & - & 15 \\
WF95-APP5 & 95 & 5 & - \\
WF85-APP5-LD10 & 85 & 5 & 10 \\
\hline
\end{tabular}

72, 94, and $177 \mathrm{ppm}$, relative to the sugar units of hemicellulose and certain lignin structures are almost absent. This feature confirms the successful removal of the polysaccharide fraction along with some lower molecular weight sulfonated lignin fragments.

${ }^{13} \mathrm{C}$ CP MAS-NMR analysis evidences that, upon dialysis, a remarkable amount of contaminants is removed from the feedstock. This outcome is in agreement with the significant weight drop (around $50 \mathrm{wt} . \%$ ) recorded after purification.

The dialysate, before and after acid hydrolysis, was analysed through HPLC in order to assess the chemical composition of the carbohydrate fraction removed from CaLS. Most of the products present in the dialysate were cyclic monosaccharides, mainly a fraction of xylose, mannose, and galactose (XMG) and a minor part of arabinose and glucose (Table S2 in Supplementary Materials). Therefore, HPLC data demonstrated that the contaminating carbohydrates present in CaLS were in a form of oligo/monosaccharides, typically belonging to hemicelluloses. Then, dialysis treatment allowed to efficiently remove hemicellulose sugars from the lignosulfonate, yielding an almost pure lignin.

The thermodegradative behaviour of CaLS and LD was investigated through TGA. The thermograms and the DTG curves are shown in Figure 1(b) while the average thermal data are reported in Table 2. The TGA plot of plain CaLS shows three weight loss steps $\left(100-160^{\circ} \mathrm{C}, 160-250^{\circ} \mathrm{C}\right.$, and $250-500^{\circ} \mathrm{C}$ temperature ranges), corresponding to different thermodegradative phenomena. On the basis of literature evidences [3,33], the first weight drop can be attributed to the loss of adsorbed water; the second to the evolution of $\mathrm{CO}, \mathrm{CO}_{2}, \mathrm{SO}_{2}$, and water due to dehydration reactions from hemicellulose and aliphatic lignin chains; and the third to the formation of $\mathrm{CO}$ and $\mathrm{CO}_{2}$ due to the decomposition of the aromatic skeleton of lignin. As observed in literature, generally, the main mass loss stage for lignosulfonates occurs between 190 and $360^{\circ} \mathrm{C}$ [34]. At higher temperatures, crosslinking between degraded products takes place, giving rise to the formation of a carbonaceous char.

The thermogram relative to $\mathrm{LD}$ is characterized by a $T_{5 \%}$ equal to $241^{\circ} \mathrm{C}$, which is remarkably higher than that of CaLS $\left(186^{\circ} \mathrm{C}\right)$. Moreover, the curve shows a single weight loss step, with a $T_{\max }$ of $304^{\circ} \mathrm{C}$ that corresponds to the third mass loss step of CaLS $\left(T_{\max 3} 300^{\circ} \mathrm{C}\right)$. The lack of the lowtemperature decomposition stages, between 100 and $250^{\circ} \mathrm{C}$, further confirms the effective removal of oligo/monosaccharides in the sample after dialysis. Therefore, TGA outcomes are in agreement with ${ }^{13} \mathrm{C}$ CP MAS-NMR and HPLC analyses that demonstrate that CaLS contains a relevant amount of hemicellulose components alongside the 


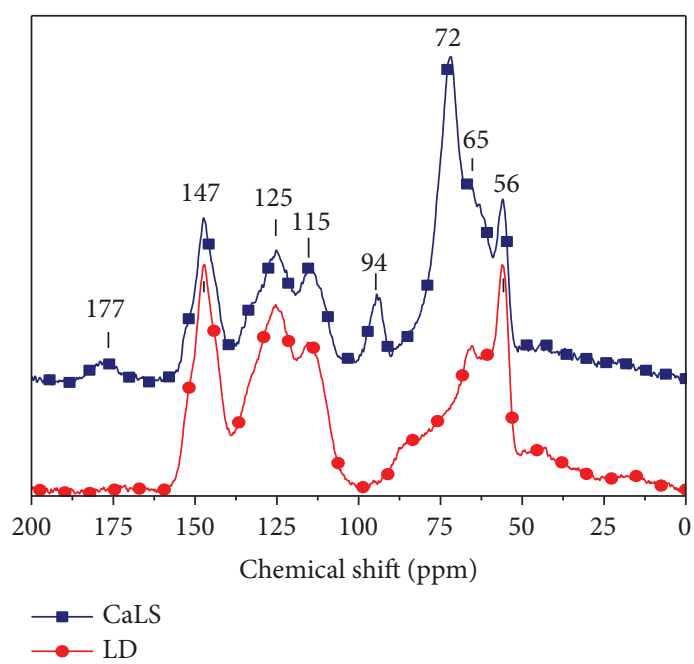

(a)

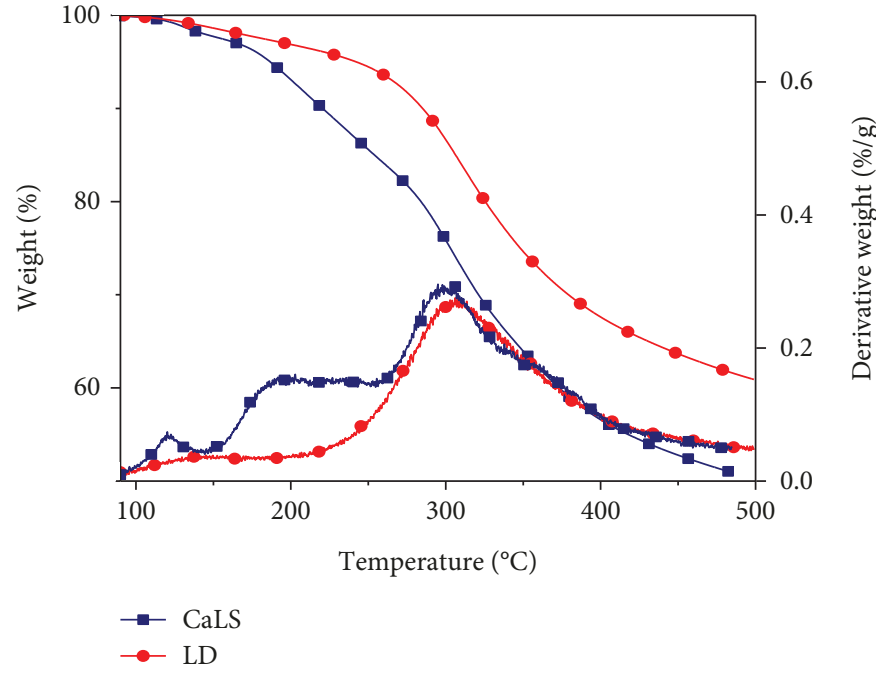

(b)

Figure 1: (a) ${ }^{13} \mathrm{C}$ CP MAS-NMR spectra of CaLS and LD. (b) TGA and DTG curves of CaLS and LD.

TABLE 2: Thermal data of CaLS and LD measured through TGA.

\begin{tabular}{lccccc}
\hline Sample & $T_{5 \%}\left({ }^{\circ} \mathrm{C}\right)$ & $\begin{array}{c}T_{\max 1} \\
\left({ }^{\circ} \mathrm{C}\right)\end{array}$ & $\begin{array}{c}T_{\max 2} \\
\left({ }^{\circ} \mathrm{C}\right)\end{array}$ & $\begin{array}{c}T_{\max 3} \\
\left({ }^{\circ} \mathrm{C}\right)\end{array}$ & $\begin{array}{c}\mathrm{Char}_{500{ }^{\circ} \mathrm{C}} \\
(\%)\end{array}$ \\
\hline CaLS & $186 \pm 3.2$ & $133 \pm 3.3$ & $200 \pm 3.5$ & $300 \pm 4.2$ & $51 \pm 1.2$ \\
LD & $241 \pm 2.4$ & - & - & $304 \pm 3.8$ & $61 \pm 1.0$ \\
\hline
\end{tabular}

lignin fraction. Moreover, TGA analysis shows that, through purification, the thermodegradative stability of the dialysed lignosulfonate is improved in a remarkable way. This finding is noteworthy for applications where good thermal performances are required.

3.2. Mechanical Properties and Morphological Analysis of WFP. Mechanical properties of the prepared WFP were evaluated by flexural tests. Table 3 lists modulus $\left(E_{f}\right)$ and strength $\left(\sigma_{f}\right)$ values measured through flexural experiments, while Fig. S2 in Supplementary Materials shows the SEM micrographs of the fracture surfaces after failure upon mechanical testing.

Compared to the plain particleboard, the samples containing solely LD show a decrease of both the flexural parameters. This outcome can be related to the lack of chemical interaction between the LD and the urea-formaldehyde resin used as binder in the particleboards. The reactivity of the binder towards LD depends on the presence, in the phenylpropanoid units of lignin, of free ortho positions close to nonetherified phenolic moieties [35]. The measurement of phenolic hydroxyl content in LD, performed by ultraviolet difference spectroscopy [36], showed a concentration below 1 wt.\% (details in Supplementary Materials, UV spectroscopy of LD, Fig. S3). This value is low if compared to other lignin products $[37,38]$ and is a hint of low reactivity. As a consequence, LD behaves as an inert or deteriorating agent towards the UF resin. A similar effect was reported for another urea-formaldehyde system modified with lignosulfo-
TABLE 3: Main flexural properties of WFP.

\begin{tabular}{lcc}
\hline Sample & $E_{f}(\mathrm{MPa})$ & $\sigma_{f}(\mathrm{MPa})$ \\
\hline WF 100 & $146 \pm 35$ & $0.50 \pm 0.21$ \\
WF95-LD5 & $115 \pm 39$ & $0.36 \pm 0.07$ \\
WF85-LD15 & $36 \pm 9$ & $0.13 \pm 0.02$ \\
WF85-APP5-LD10 & $121 \pm 28$ & $0.49 \pm 0.09$ \\
WF95-APP5 & $233 \pm 72$ & $0.88 \pm 0.16$ \\
\hline
\end{tabular}

nates [39]. Conversely, an improvement in mechanical performances was recorded for the WF95-APP5 composite, which was modified with APP. The enhancement was detected on modulus and strength as well, and it can be attributed to the reinforcing action of APP towards UF resin due to the good physical interactions between the two components [40]. The good compatibility between APP and UF resin has also been used in other FR formulations [41].

Finally, the WF85-APP5-LD10 sample, containing both lignosulfonates and APP, shows no variation or only a slight decrease of the flexural parameters with respect to the plain WFP. These results are probably related to the presence of the APP additive that partly compensates the detrimental action of the lignin component.

3.3. Thermal Analysis and Fire Behaviour of WFP. The thermodegradative behaviour of WFP was assessed through TGA measurements performed in nitrogen. The TGA curves, along with the DTG graphs, are reported in Figure 2, while thermal parameters are listed in Table 4.

From TGA curves, plain WFP exhibited a single-step weight loss (Figure $2(\mathrm{a})$ ) with a $T_{5 \%}$ of $260^{\circ} \mathrm{C}$, a $T_{\max }$ equal to $366^{\circ} \mathrm{C}$, and a $\mathrm{Char}_{700^{\circ} \mathrm{C}}$ of $22 \%$. The addition of $\mathrm{LD}$ to the particleboards produced nearly no effect on the thermal parameters. As for the APP-based specimens, both of them exhibit a small peak in their DTG curves below $200^{\circ} \mathrm{C}$, related 


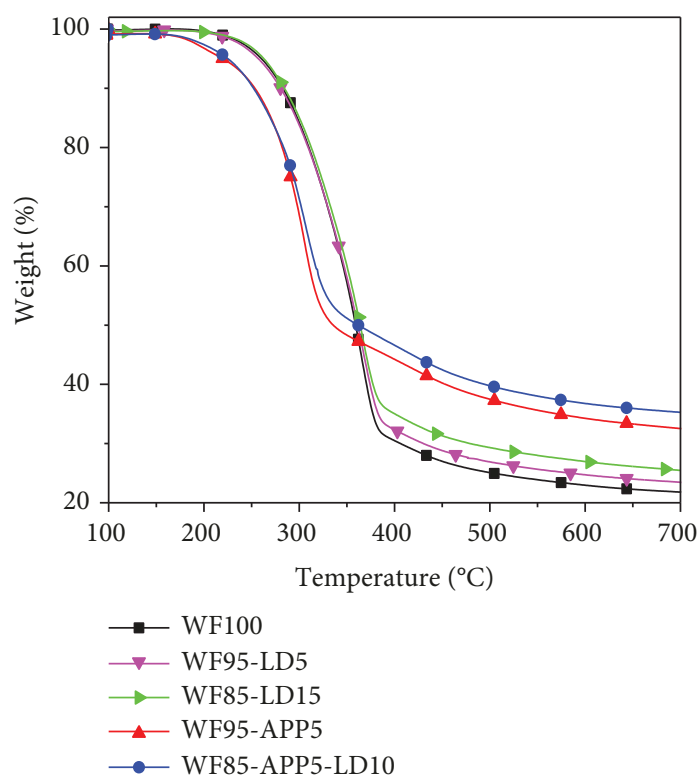

(a)

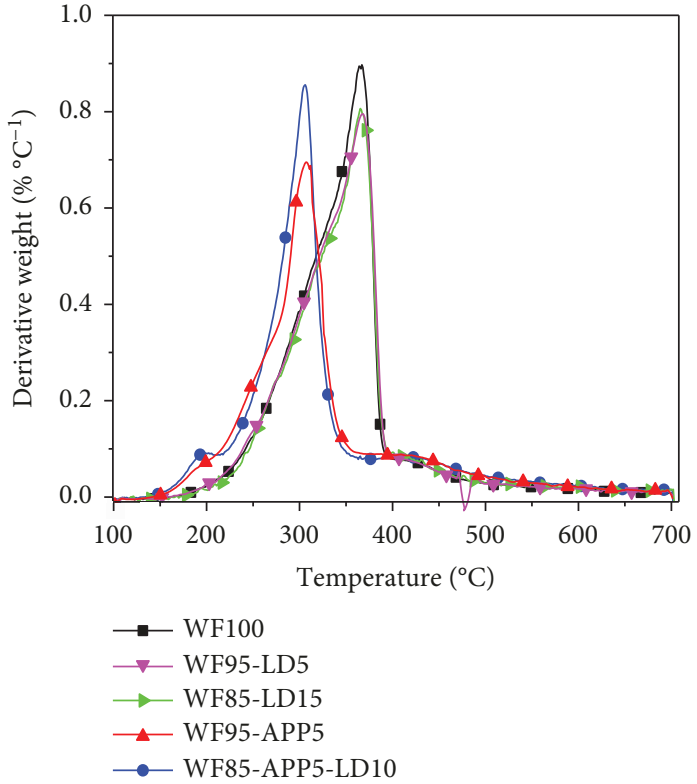

(b)

FIgURe 2: (a) TGA and (b) DTG curves of WFP.

TABLE 4: Thermal data of WFP measured through TGA.

\begin{tabular}{lccc}
\hline Sample & $T_{5 \%}\left({ }^{\circ} \mathrm{C}\right)$ & $T_{\max }\left({ }^{\circ} \mathrm{C}\right)$ & Char $_{700^{\circ} \mathrm{C}}(\%)$ \\
\hline WF100 & 260 & 366 & 22 \\
WF95-LD5 & 257 & 368 & 23 \\
WF85-LD15 & 262 & 367 & 25 \\
WF95-APP5 & 220 & 306 & 32 \\
WF85-APP5-LD10 & 225 & 306 & 35 \\
\hline
\end{tabular}

to the decomposition of thermally unstable structural groups of APP [42], followed by the main degradative phenomenon. The WF95-APP5 particleboard showed a $T_{5 \%}$ of $220^{\circ} \mathrm{C}$ and a $T_{\max }$ equal to $306^{\circ} \mathrm{C}$. These values appeared to be much lower if compared to the WF100 sample. Such a result was ascribed to a noticeable decrease in thermal resistance of the material that can be related to the decomposition of APP. Conversely, the char-forming capability of the WF95-APP5 particleboard resulted considerably higher, with a $\mathrm{Char}_{700^{\circ} \mathrm{C}}$ equal to $32 \%$. WF85-APP5-LD10 showed a moderate increase of $T_{5 \%}$ $\left(225^{\circ} \mathrm{C}\right)$ and char yield (35\%) comparable to the composite modified with only APP.

The efficiency of LD as a FR, alone or along with the commercial fire retardant APP, was studied by cone calorimeter tests on WFP. The selection of the thermal attack used in the analysis is a critical point in the research on fire retardancy [43]. In this work, an average value $\left(35 \mathrm{~kW} \mathrm{~m}^{-2}\right)$ with respect to those typically used in this kind of studies was chosen, according to ISO/TS 5660-3:2012. Figure 3 shows the visual appearance of some of the particleboards prior to the cone calorimeter tests (a) and after the combustion process (b).

Prior to the test, the samples appeared as compact and sufficiently homogeneous plates. By a visual examination, the reference WF100 and the lignosulfonate-modified particleboards were brittle and dusty, while the samples containing APP were more compact and stiffer, with a more brilliant colour than the others. After the combustion tests, WF100 and particleboards filled with LD showed a carbonized aspect with several cracks and no morphological differences were noticed. Conversely, the fire-retarded samples filled with APP appeared black, solid, and characterized by a thick and compact structure.

The main average cone calorimeter test results are reported in Table 5, while Figure 4 shows the heat release rate (HRR), the total heat release (THR), and the total smoke production (TSP) as a function of combustion time.

HRR is one of the most important parameters for the characterization of material flame performance because it expresses the intensity of a fire. Typically, wood burns according to the HRR curves shown in Figure 4(a). After an initial heating induction time, a first peak is detected. It is related to ignition which consists in heating the sample in order to generate the emission of pyrolytic gases that are ignited by an external spark. Combustion starts with heat production and further emission of pyrolytic gases for ongoing sustenance of combustion. After this first stage, an insulating char layer forms on the surface of the material that prevents the evolution of volatiles so the combustion process slows down. The second peak of the graphs is generated by the breakage of the insulating layer that allows the emission of pyrolytic volatiles. From their combustion, more heat is generated, and the second HRR peak appears [16]. Two important features of the plots are ignition time $\left(t_{\mathrm{ig}}\right)$, which is the time for the ignition phenomenon to occur, and the height of the second peak $\left(\mathrm{HRR}_{\max }\right)$. As for the $t_{\mathrm{ig}}$, all the tested samples were characterized by values in the range of 41-49 sec and the effect of the additive resulted negligible. 
WF100
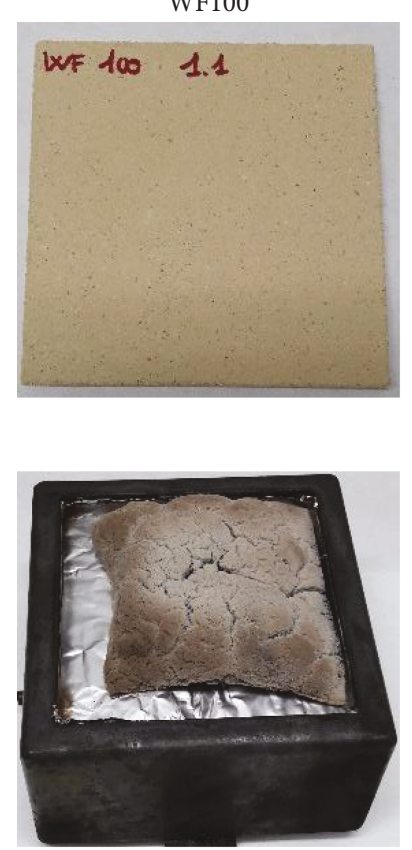

WF95-LD5

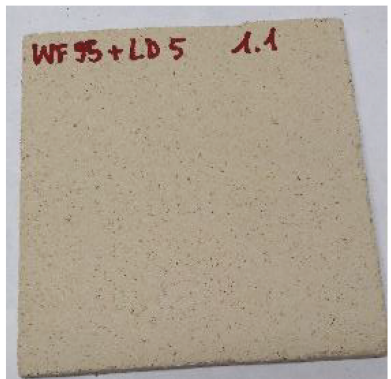

(a)

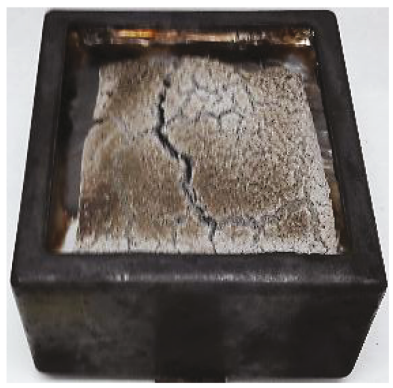

(b)
WF85-APP5-LD10
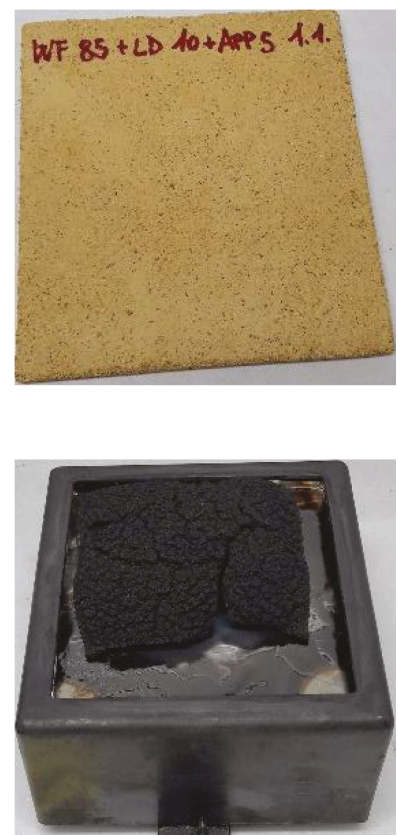

FIGURE 3: Photo of some of the prepared WFP, prior to the cone calorimeter test (a) and after combustion (b).

TABLE 5: Average results obtained from cone calorimeter characterization of WFP.

\begin{tabular}{|c|c|c|c|c|c|}
\hline Sample & $\begin{array}{l}\mathrm{HRR}_{\max } \\
\left(\mathrm{kW} \mathrm{m}^{-2}\right)\end{array}$ & $\begin{array}{c}\text { THR } \\
\left(\mathrm{MJ} \mathrm{m}^{-2}\right)\end{array}$ & $\begin{array}{l}\text { ML } \\
(\%)\end{array}$ & $\begin{array}{l}\text { TSP } \\
\left(\mathrm{m}^{2}\right)\end{array}$ & $\begin{array}{c}\text { SEA } \\
\left(\mathrm{m}^{2} \mathrm{~kg}^{-1}\right)\end{array}$ \\
\hline WF100 & $310.8 \pm 11.9$ & $53.2 \pm 2.7$ & $91.4 \pm 1.5$ & $1.8 \pm 0.1$ & $44.7 \pm 3.9$ \\
\hline WF95-LD5 & $268.9 \pm 47.5$ & $54.7 \pm 6.0$ & $93.3 \pm 2.3$ & $1.7 \pm 0.3$ & $50.7 \pm 5.6$ \\
\hline WF85-LD15 & $230.5 \pm 20.4$ & $50.8 \pm 2.6$ & $87.3 \pm 2.1$ & $1.3 \pm 0.1$ & $34.4 \pm 10.9$ \\
\hline WF95-APP5 & $228.0 \pm 7.1$ & $41.5 \pm 2.9$ & $88.7 \pm 2.1$ & $0.5 \pm 0.0$ & $14.9 \pm 5.5$ \\
\hline WF85-APP5-LD10 & $187.6 \pm 11.0$ & $40.0 \pm 1.1$ & $83.6 \pm 2.2$ & $0.5 \pm 0.1$ & $16.2 \pm 3.4$ \\
\hline
\end{tabular}

$\mathrm{HRR}_{\max }$, conversely, is strongly influenced by the composition of the compound (Table 5). Compared with the reference WF100, the samples modified solely with LD were characterized by a decrease in $\mathrm{HRR}_{\max }$ (Table 5) achieving a $25 \%$ reduction in the case of WF85-LD15. This result suggests a moderate fire-suppressing effect ascribed to the LD modifier towards the WFP. As for the THR graph (Figure 4(b)) that represents the area below the HRR plot, it was observed that the addition of LD produced nearly no effect on THR, with respect to the reference WF100.

Mass loss (ML) is another important parameter obtained from the cone calorimeter tests. It is related to the charforming capability of the material, as the lower the mass loss the higher the char residue during combustion. As shown in Table 5, the addition of LD in the formulation produces a slight effect on ML with respect to the plain WFP.

Smoke performance of fire-retarded materials is a significant index in fire safety. It is measured by the total smoke production (TSP) which is reported in Figure 4(c). As it is evident from Table 5, the particleboard containing $15 \mathrm{wt} . \%$
LD released less smoke during combustion than the reference. This outcome is positive in terms of fire retardancy. Most traditional fire retardants, indeed, besides retarding fire evolution also decrease smoke production. Another index of the smoke emission is the specific extinction area, referred as SEA. It is a measure of the smoke yield: the higher the SEA, the more the amount of smoke emitted per $\mathrm{kg}$ of sample [44]. By comparing the SEA of neat WF100 with that of LD-modified particleboards, it is noticed that $15 \mathrm{wt} . \%$ LD caused a decrease in this parameter (Table 5).

In order to assess the role of $\mathrm{LD}$ in the presence of a conventional FR, the flame performances of a particleboard sample containing 10\% LD and 5\% APP, namely, WF85APP5-LD10, was investigated through a cone calorimeter. Therefore, the combined effect of LD and APP was studied. For comparison, a sample containing solely $5 \%$ of APP was also tested. The fire behaviour of a particleboard at higher APP content (15\%) was tested as well. Results are reported in Supplementary Materials, fire behaviour of WFP. 


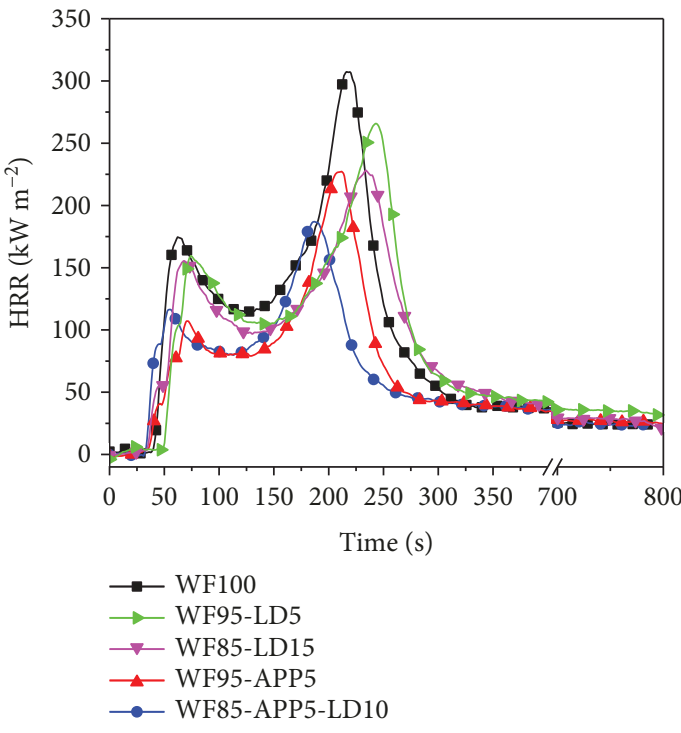

(a)

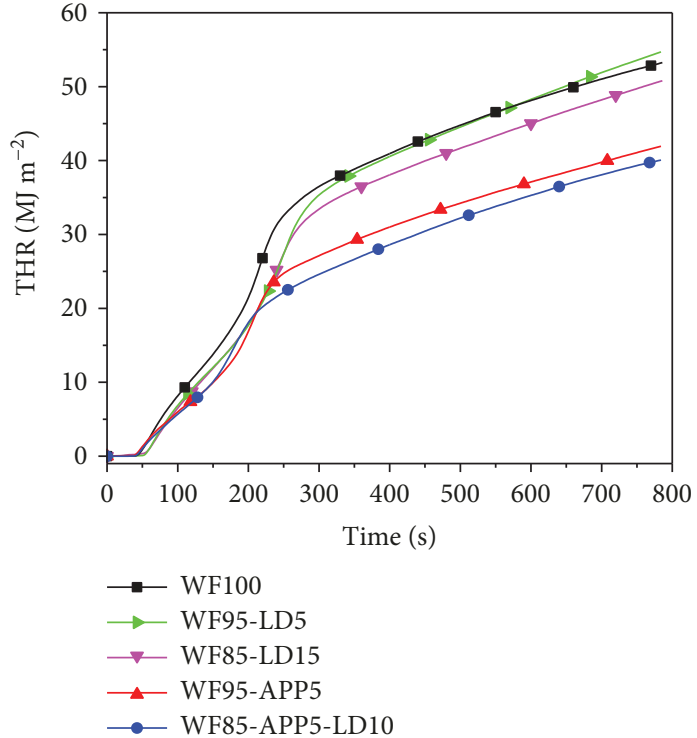

(b)

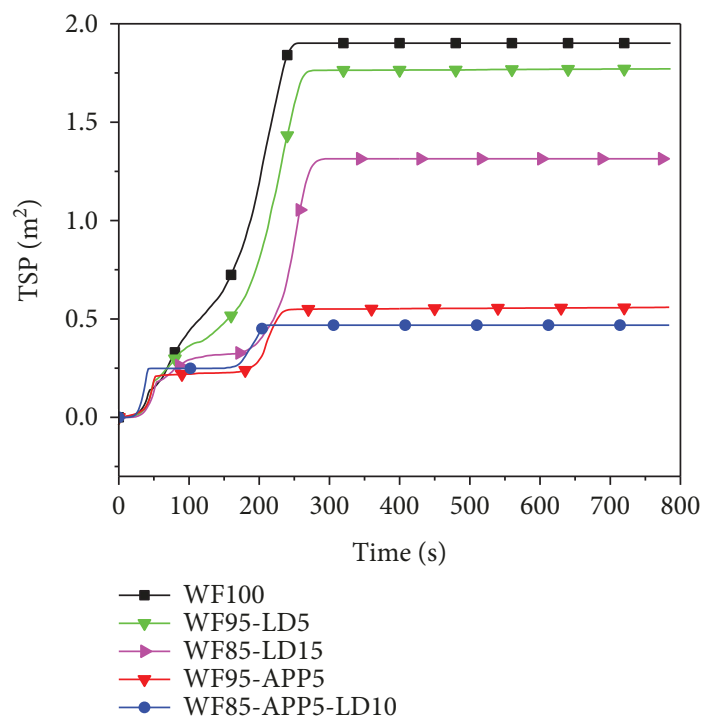

(c)

FIgURE 4: (a) HRR, (b) THR, and (c) TSP curves vs. combustion time of prepared WFP.

As for $\mathrm{HRR}_{\max }$, the WF85-APP5-LD10 composite showed a $20 \%$ drop compared to its reference WF95-APP5, resulting in an overall $40 \%$ decrease with respect to WF100. A similar behaviour was found regarding ML, which is related to the char-forming capability of the material. In this case, the addition of solely $5 \mathrm{wt} . \%$ APP, compared to WF100, produced only a weak effect on ML. Conversely, the use of 10 wt.\% of LD along with APP in WF85-APP5-LD10 brought a more pronounced decrease of this parameter and, consequently, a higher char yield. APP, indeed, when exposed to a combustion, starts to decompose into poly(phosphoric acid) and ammonia. Poly(phosphoric acid) reacts with hydroxyl groups to form an unstable phosphate ester. When the phosphate ester starts to dehydrate, a carbonaceous residue forms, thus creating the so-called charring effect. The carbon foam acts as an insulating layer, thus pre- venting the development of the combustion and decomposition of the material [14]. This charring mechanism appears to be more effective when lignosulfonates are introduced in the formulation along with APP. A similar outcome has been found by Li et al. [27], who claim that APP acts as a charring promoter towards lignin which helps the formation of a char layer [15]. APP, indeed, is able to form char through the cleavage of lignin $\mathrm{C}-\mathrm{O}$ bonds, and its decomposition product, polyphosphoric acid, catalyses crosslinking reactions [27]. However, it should be pointed out that this charring effect can be also attributed to the reaction of $\mathrm{C}-\mathrm{OH}$ groups of lignin and polyphosphoric acid moieties produced from APP during combustion, with the production of phosphoric esters that improve the char-forming performances [23, 45].

As regards the smoke emission, the TSP and SEA data (Figure 4(c) and Table 5) evidenced that the addition of 
$5 \mathrm{wt} . \%$ APP produced a noticeable drop of both parameters with respect to WF100 and the LD-modified WFPs. The introduction of LD along with APP did not result in a further decrease of the smoke emission indexes.

Overall, a general improvement of LD-modified WFP fire performances, with respect to WF100, was evidenced. In some cases, this effect was enhanced if APP was also introduced into the formulation.

\section{Conclusions}

In conclusion, a dialysed waste lignosulfonate was used as a low-cost additive to improve fire behaviour of urea formaldehyde WFP. Dialysis proved to be a simple and efficient technique for the purification of CaLS that allowed to recover a polysaccharide free LD. The latter, when introduced into the WFP, showed a fire-retardant activity, by reducing HRR, THR, and TSP parameters and increasing char formation. The lignin aromatic component acts as a char former, which represents a well-known mechanism in fire retardant additives. Char, indeed, reduces the combustion rate of wood hindering oxygen diffusion to the burning zone. This fire behaviour improvement is magnified if a relatively small amount of APP is added to the particleboard along with $\mathrm{LD}$. The interaction between the two components is probably based on the catalytic crosslinking action of APP towards $\mathrm{LD}$ or to the reaction of $\mathrm{C}-\mathrm{OH}$ groups of lignin and polyphosphoric acid moieties produced from APP during combustion. Overall, this work demonstrates that the use of unmodified lignosulfonate represents an attractive and costeffective option to improve fire retardancy of WFP, widely used in furniture or green building applications.

\section{Data Availability}

The data used to support the findings of this study are included within the article.

\section{Disclosure}

Part of this work was previously presented at the XXIII meeting of Associazione Italiana di Scienza e Tecnologia delle Macromolecole (AIM), held September 9-12, 2018, in Catania, Italy.

\section{Conflicts of Interest}

The authors declare that there is no conflict of interest regarding the publication of this paper.

\section{Acknowledgments}

The authors earnestly thank Dr. Mattia Gelosia for performing High-Performance Liquid Chromatography analysis. Financial support from the CyberSort project, Accordo Quadro CNR-Regione Lombardia, is acknowledged.

\section{Supplementary Materials}

Additional Supplementary Materials may be found in the online version of this article. They concern the morphological and chemical analysis of lignosulfonates along with the morphological characterization of WFP samples. (Supplementary Materials)

\section{References}

[1] O. Y. Abdelaziz and C. P. Hulteberg, "Physicochemical characterisation of technical lignins for their potential valorisation," Waste and Biomass Valorization, vol. 8, no. 3, pp. 859-869, 2017.

[2] M. E. Eugenio, J. M. Carbajo, M. C. Terrón, A. E. González, and J. C. Villar, "Bioremediation of lignosulphonates by lignin-degrading basidiomycetous fungi," Bioresource Technology, vol. 99, no. 11, pp. 4929-4934, 2008.

[3] A. P. Lemes, M. A. Soto-Oviedo, W. R. Waldman, L. H. Innocentini-Mei, and N. Duràn, "Effect of lignosulfonate on the thermal and morphological behavior of poly(3hydroxybutyrate-co-3-hydroxyvalerate)," Journal of Polymers and the Environment, vol. 18, no. 3, pp. 250-259, 2010.

[4] Y. Matsushita and S. Yasuda, "Preparation and evaluation of lignosulfonates as a dispersant for gypsum paste from acid hydrolysis lignin," Bioresource Technology, vol. 96, no. 4, pp. 465-470, 2005.

[5] S. A. Gundersen and J. Sjoblom, "High- and low-molecularweight lignosulfonates and Kraft lignins as oil/water-emulsion stabilizers studied by means of electrical conductivity," Colloid \& Polymer Science, vol. 277, no. 5, pp. 462-468, 1999.

[6] T. Aro and P. Fatehi, "Production and application of lignosulfonates and sulfonated lignin," ChemSusChem, vol. 10, no. 9, pp. 1861-1877, 2017.

[7] S. G. Santos, A. P. Marques, D. L. D. Lima, D. V. Evtuguin, and V. I. Esteves, "Kinetics of eucalypt lignosulfonate oxidation to aromatic aldehydes by oxygen in alkaline medium," Industrial and Engineering Chemistry Research, vol. 50, no. 1, pp. 291298,2011

[8] M. B. Hocking, "Vanillin: synthetic flavoring from spent sulfite liquor," Journal of Chemical Education, vol. 74, no. 9, p. 1055, 1997.

[9] Y. Pan, J. Zhan, H. Pan et al., "Effect of fully biobased coatings constructed via layer-by-layer assembly of chitosan and lignosulfonate on the thermal, flame retardant, and mechanical properties of flexible polyurethane foam," ACS Sustainable Chemistry \& Engineering, vol. 4, no. 3, pp. 1431-1438, 2015.

[10] L. C. Morais, A. A. S. Curvelo, and M. D. Zambon, "Thermoplastic starch-lignosulfonate blends. 1. factorial planning as a tool for elucidating new data from high performance sizeexclusion chromatography and mechanical tests," Carbohydrate Polymers, vol. 62, no. 2, pp. 104-112, 2005.

[11] C. Galán-Marín, C. Rivera-Gómez, and F. Bradley, "Ultrasonic, molecular and mechanical testing diagnostics in natural fibre reinforced, polymer-stabilized earth blocks," International Journal of Polymer Science, vol. 2013, Article ID 130582, 10 pages, 2013.

[12] K. G. Satyanarayana, G. G. C. Arizaga, and F. Wypych, "Biodegradable composites based on lignocellulosic fibers-An overview," Progress in Polymer Science, vol. 34, no. 9, pp. 982-1021, 2009. 
[13] D. Y. Wang, E. Naderi Kalali, and J. Croyal, "Fire retardant wood polymer composites: preparation, flammability, mechanical properties," in Proceedings of the 1st European Workshop Fire Safety of Green Buildings, pp. 33-35, Berlin, Germany, 2015.

[14] L. V. P. Pin, Z. Wang, K. Hu, and W. Fan, "Flammability and thermal degradation of flame retarded polypropylene composites containing melamine phosphate and pentaerythritol derivatives," Polymer Degradation and Stability, vol. 90, no. 3, pp. 523-534, 2005.

[15] X. Chen, Y. Jiang, and C. Jiao, "Smoke suppression properties of ferrite yellow on flame retardant thermoplastic polyurethane based on ammonium polyphosphate," Journal of Hazardous Materials, vol. 266, pp. 114-121, 2014.

[16] D. C. O. Marney, L. J. Russell, and R. Mann, "Fire performance of wood (Pinus radiata) treated with fire retardants and a wood preservative," Fire and Materials, vol. 32, no. 6, pp. 357-370, 2008.

[17] R. H. White, "Oxygen index evaluation of fire-retardanttreated wood," Wood Science, vol. 12, pp. 113-121, 1979.

[18] R. Hashim, O. Sulaiman, R. N. Kumar, P. F. Tamyez, R. J. Murphy, and Z. Ali, "Physical and mechanical properties of flame retardant urea formaldehyde medium density fiberboard," Journal of Materials Processing Technology, vol. 209, no. 2, pp. 635-640, 2009.

[19] O. Grexa, F. Poutch, D. Manikova, H. Martvonova, and A. Bartekova, "Intumescence in fire retardancy of lignocellulosic panels," Polymer Degradation and Stability, vol. 82, no. 2, pp. 373-377, 2003.

[20] Z. A. Nagieb, M. A. Nassar, and M. G. el-Meligy, "Effect of addition of boric acid and borax on fire-retardant and mechanical properties of urea formaldehyde saw dust composites," International Journal of Carbohydrate Chemistry, vol. 2011, Article ID 146763, 6 pages, 2011.

[21] G. Liu, W. Chen, and J. Yu, "A novel process to prepare ammonium polyphosphate with crystalline form ii and its comparison with melamine polyphosphate," Industrial and Engineering Chemistry Research, vol. 49, no. 23, pp. 1214812155, 2010.

[22] C. Reti, M. Casetta, S. Duquesne, S. Bourbigot, and R. Delobel, "Flammability properties of intumescent pla including starch and lignin," Polymers for Advanced Technologies, vol. 19, no. 6, pp. 628-635, 2008.

[23] L. Costes, F. Laoutid, M. Aguedo et al., "Phosphorus and nitrogen derivatization as efficient route for improvement of lignin flame retardant action in PLA," European Polymer Journal, vol. 84, pp. 652-667, 2016.

[24] L. Ferry, G. Dorez, A. Taguet, B. Otazaghine, and J. M. LopezCuesta, "Chemical modification of lignin by phosphorus molecules to improve the fire behavior of polybutylene succinate," Polymer Degradation and Stability, vol. 113, pp. 135-143, 2015.

[25] L. Liu, M. Qian, P.’. Song, G. Huang, Y. Yu, and S. Fu, “Fabrication of green lignin-based flame retardants for enhancing the thermal and fire retardancy properties of polypropylene/wood composites," ACS Sustainable Chemistry \& Engineering, vol. 4, no. 4, pp. 2422-2431, 2016.

[26] 2018, https://www.alibaba.com/product-detail/calcium-lignosul fonate-powder_62035133245.html?spm=a2700.7724838.2017115. 50.2dd8752bWG3JOB\&s=phttps://www.alibaba.comhttps://www .alibaba.com/product-detail/retardant-ammonium-polyphos phate_60613838038.html?spm=a2700.7724838.2017115.107.7901 42b2LXvnaJ.
[27] B. Li, X. Zhang, and R. Su, "An investigation of thermal degradation and charring of larch lignin in the condensed phase: the effects of boric acid, guanyl urea phosphate, ammonium dihydrogen phosphate and ammonium polyphosphate," Polymer Degradation and Stability, vol. 75, no. 1, pp. 35-44, 2002.

[28] H. Struszczyk, "Modification of Lignins. III. Reaction of Lignosulfonates with Chlorophosphazenes," Journal of Macromolecular Science: Part A - Chemistry, vol. 23, no. 8, pp. 973-992, 1986.

[29] Y. Yu, S. Fu, P.’. Song et al., "Functionalized lignin by grafting phosphorus-nitrogen improves the thermal stability and flame retardancy of polypropylene," Polymer Degradation and Stability, vol. 97, no. 4, pp. 541-546, 2012.

[30] L. Liu, G. Huang, P. Song, Y. Yu, and S. Fu, "Converting industrial alkali lignin to biobased functional additives for improving fire behavior and smoke suppression of polybutylene succinate," ACS Sustainable Chemistry \& Engineering, vol. 4, no. 9, pp. 4732-4742, 2016.

[31] L. Verdolotti, M. Oliviero, M. Lavorgna et al., "On revealing the effect of alkaline lignin and ammonium polyphosphate additives on fire retardant properties of sustainable zeinbased composites," Polymer Degradation and Stability, vol. 134, pp. 115-125, 2016.

[32] A. Sluiter, B. Hames, R. Ruiz et al., Determination of structural carbohydrates and lignin in biomass, Laboratory Analytical Procedure, 2008, http://www.nrel.gov/biomass/ analyticalprocedures.html.

[33] Z. Luo, S. Wang, and X. Guo, "Selective pyrolysis of Organosolv lignin over zeolites with product analysis by TG-FTIR," Journal of Analytical and Applied Pyrolysis, vol. 95, pp. 112117, 2012.

[34] V. S. Ramachandran and R. F. Feldman, "Adsorption of calcium lignosulfonate on tricalcium aluminate and its hydrates in a non-aqueous medium," Cement Technology, vol. 2, no. 4, pp. 121-129, 1971.

[35] A. Duval and M. Lawoko, "A review on lignin-based polymeric, micro- and nano-structured materials," Reactive \& Functional Polymers, vol. 85, pp. 78-96, 2014.

[36] S. Angelini, D. Ingles, M. Gelosia et al., "One-pot lignin extraction and modification in $\gamma$-valerolactone from steam explosion pre-treated lignocellulosic biomass," Journal of Cleaner Production, vol. 151, pp. 152-162, 2017.

[37] N.-E. El Mansouri and J. Salvadó, "Structural characterization of technical lignins for the production of adhesives: Application to lignosulfonate, kraft, soda-anthraquinone, organosolv and ethanol process lignins," Industrial Crops and Products, vol. 24, no. 1, pp. 8-16, 2006.

[38] M. V. Alonso, J. J. Rodríguez, M. Oliet, F. Rodríguez, J. Garcia, and M. A. Gilarranz, "Characterization and structural modification of ammonic lignosulfonate by methylolation," Journal of Applied Polymer Science, vol. 82, no. 11, pp. 2661-2668, 2001.

[39] A. M. Ferreira, J. Pereira, M. Almeida et al., "Effect of spent sulfite liquor on urea-formaldehyde resin performance," Journal of Applied Polymer Science, vol. 136, no. 17, 2019.

[40] Y. Xiang, X. Ru, J. Shi et al., "Granular, slow-release fertilizer from urea-formaldehyde, ammonium polyphosphate, and amorphous silica gel: a new strategy using cold extrusion," Journal of Agricultural and Food Chemistry, vol. 66, no. 29, pp. 7606-7615, 2018. 
[41] B. Cao, X. Gu, X. Song et al., "The flammability of expandable polystyrene foams coated with melamine modified urea formaldehyde resin," Journal of Applied Polymer Science, vol. 134, no. 5, article 44423, 2017.

[42] J. Zhou, L. Yang, X. Wang, Q. Fu, Q. Sun, and Z. Zhang, "Microencapsulation of APP-I and influence of microencapsulated APP-I on microstructure and flame retardancy of PP/APP-I/PER composites," Applied Polymer Science, vol. 129, no. 1, pp. 36-46, 2013.

[43] V. Babrauskas, Fire and Materials, vol. 19, no. 6, pp. 243-252, 1995.

[44] B. A. L. Ostman, Heat Release in Fires, V. Babrauskas and S. J. Grayson, Eds., Chapman \& Hall, London, UK, 1995.

[45] P. Rybiński, B. Syrek, M. Masłowski et al., "Influence of lignocellulose fillers on properties natural rubber composites," Journal of Polymers and the Environment, vol. 26, no. 6, pp. 2489-2501, 2018. 


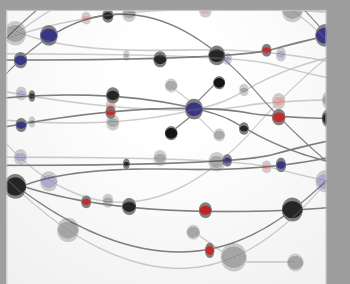

The Scientific World Journal
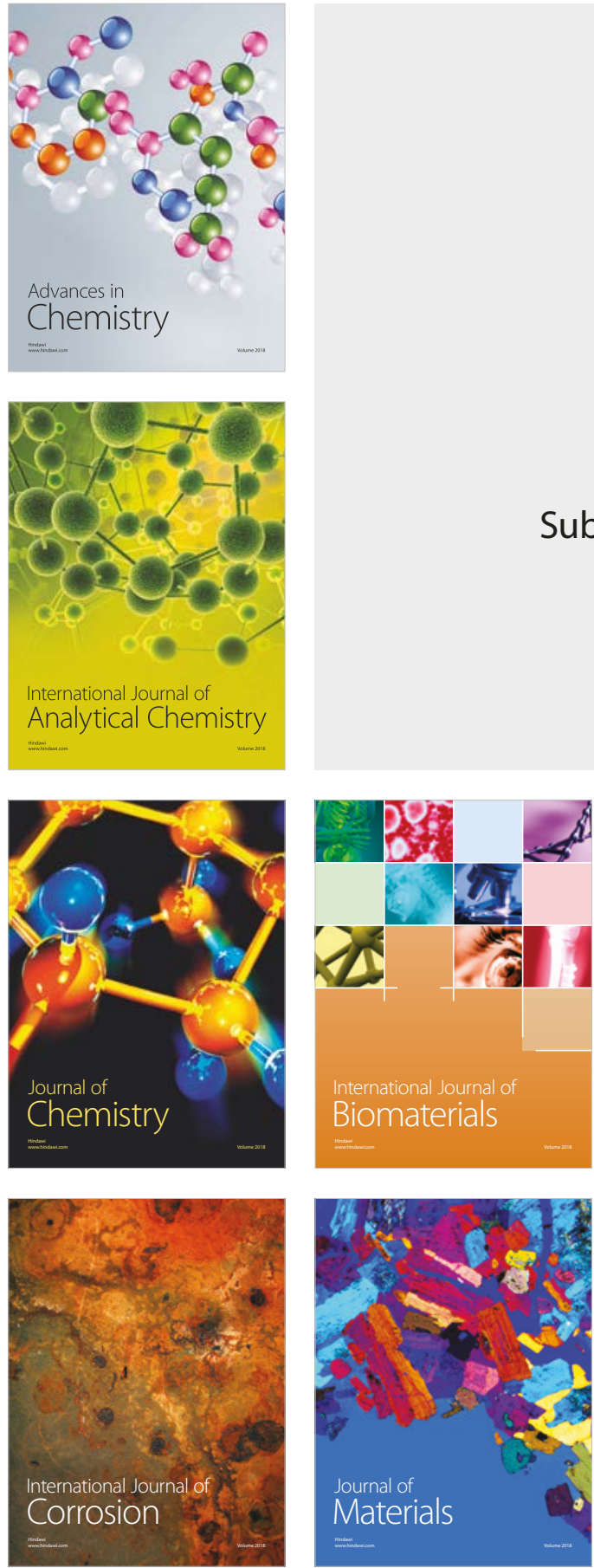

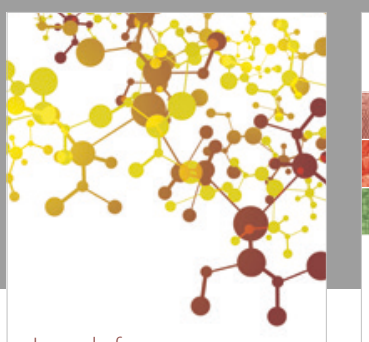

Journal of

Applied Chemistry
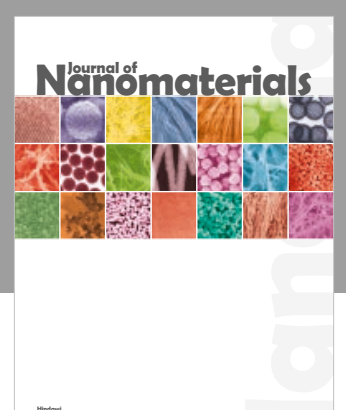

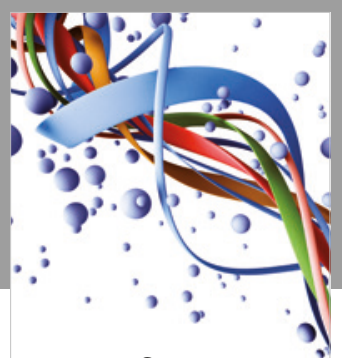

Scientifica

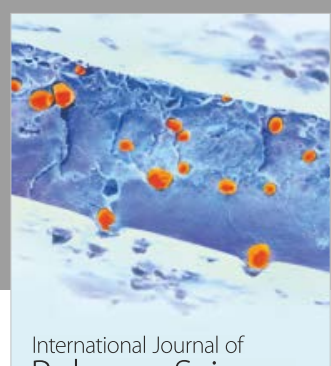

Polymer Science

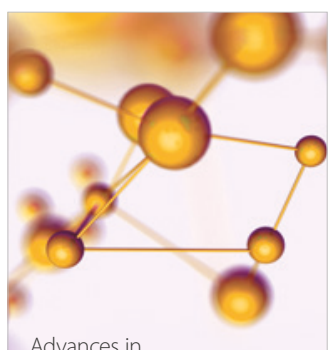

Physical Chemistry
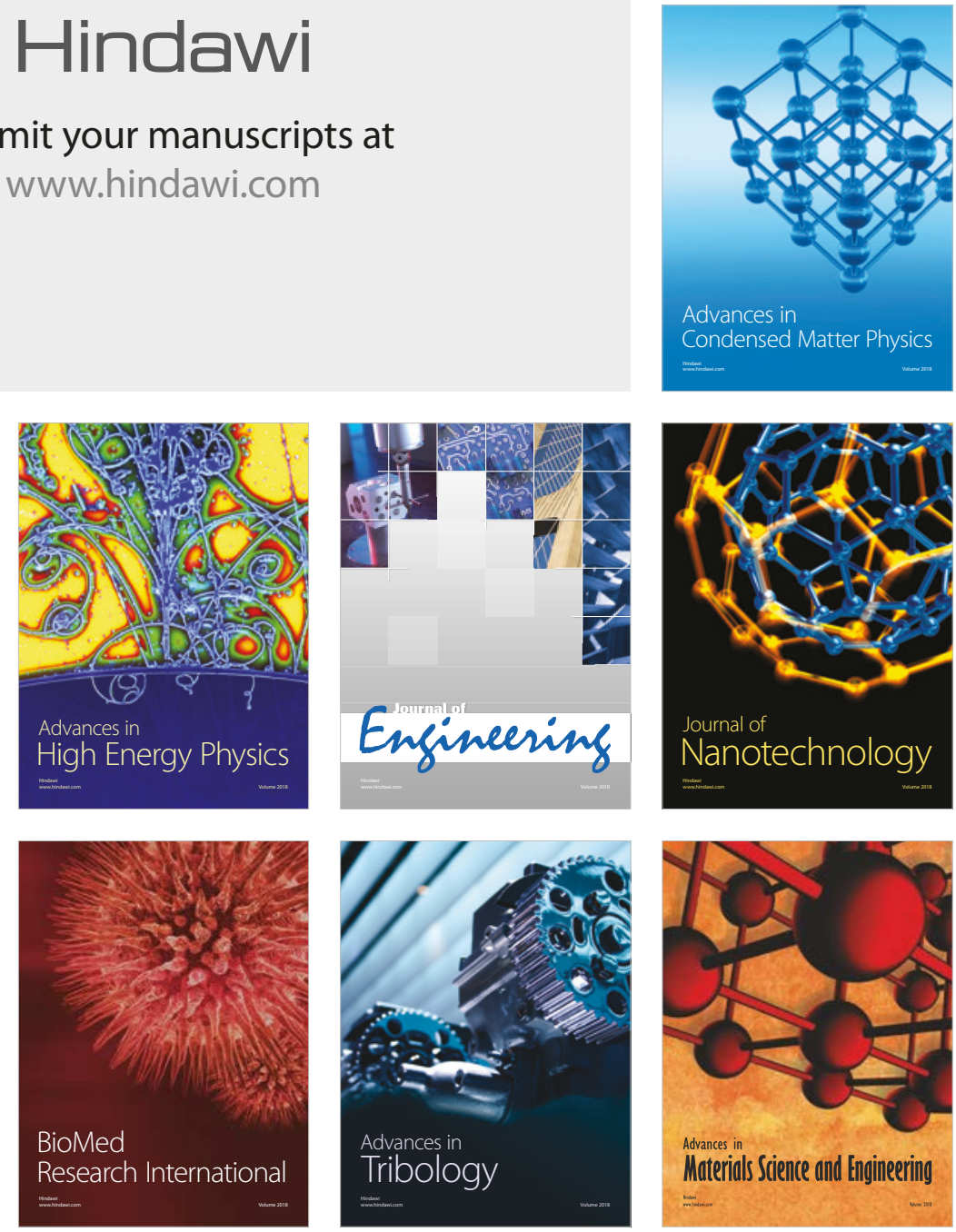